Chronic Obstructive Pulmonary Diseases: Journal of the COPD Foundation

\author{
Journal Club
}

\title{
Journal Club: Do Inhaled Corticosteroids Reduce All-Cause Mortality in Chronic Obstructive Pulmonary Disease? What is the Latest Evidence?
}

\author{
Ron Balkissoon, MD, MSc, DIH, FRCPC ${ }^{1}$
}

\begin{abstract}
Abbreviations: chronic obstructive pulmonary disease, COPD; Towards a Revolution in COPD Health study, TORCH; fluticasone propionate, FP; salmeterol, SAL; Study to Understand Mortality and Morbidity in COPD, SUMMIT; fluticasone furoate, FF; vilanterol, VI; Investigating New Standards for Prophylaxis in Reducing Exacerbations study, INSPIRE; inhaled corticosteroid, ICS; long-acting beta2agonist, LABA; long-acting muscarinic antagonist, LAMA; bipropionate, BDP; formaterol fumorate, FORF; glycopyrronium bromide, G; InforMing the Pathway of COPD Treatment, IMPACT; Efficacy and Safety of Triple Therapy in Obstructive Lung Disease, ETHOS; forced expiratory volume in 1 second, FEV $\mathbf{1}$; umeclidinium, UMEC; budesonide, BUD

Citation: Balkissoon R. Journal club- Do inhaled corticosteroids reduce all-cause mortality in chronic obstructive pulmonary disease? What is the latest evidence? Chronic Obstr Pulm Dis. 2021;8(1):177-184. doi: https://doi.org/10.15326/jcopdf.2020.0196
\end{abstract}

1 Denver, Colorado

Address correspondence to:

Ron Balkissoon, MD, MSc, DIH, FRCPC

balkissoonr@njhealth.org

\section{Keywords}

COPD; chronic obstructive pulmonary diseases; inhaled corticosteroids; all-cause mortality

\section{Introduction}

Chronic obstructive pulmonary disease (COPD) has been recognized as the third leading cause of death globally for the past several years. The majority of deaths in COPD patients are related to exacerbations, cardiovascular events and cancer. ${ }^{1}$ To date, aside from supplemental oxygen for those with severe persistent hypoxemia, smoking cessation, and lung volume reduction surgery for a subset of patients with emphysema, there have been no other interventions, particularly pharmacologic interventions, that have been proven to reduce mortality. There remains an ongoing debate about whether inhaled corticosteroids might actually have an impact on mortality.

The Towards a Revolution in COPD Health (TORCH) study $^{2}$ with 6112 participants compared fluticasone propionate (FP) $500 \mu \mathrm{g} / \mathrm{salmeterol}$ (SAL) $50 \mu \mathrm{g}$ to placebo (allowed to use short-acting bronchodilators) and demonstrated a $17.5 \%$ (95\% CI, -0.2 to 31.9 ) reduction in the hazard of death in the (FP/SAL) group compared to placebo $(P=0.052)$. It missed reaching statistical significance in part due to a prespecified interim analysis that raised the threshold for significance.

Based on the mortality data from the TORCH trial that showed similar reductions in cardiovascular and respiratory-related deaths, the Study to Understand Morbidity and Mortality in COPD (SUMMIT) trial ${ }^{3}$ compared fluticasone furoate(FF) $100 \mu \mathrm{g} /$ vilanterol (VI) $25 \mu \mathrm{g}$ to placebo and demonstrated a $12.2 \%$ reduction in the risk of death (hazard ratio 0.88 (95\%CI, 0.74 to $1.04 ; P=0.137$ ). The study enrolled a group of mild COPD patients who were required to have or be at high risk for cardiovascular disease. The study was likely underpowered due to an overestimate of the assumed reduction in mortality for a group of patients with relatively mild COPD.

The Investigating New Standards for Prophylaxis in Reducing Exacerbations study (INSPIRE) ${ }^{4}$ compared FP/SAL to tiotropium and revealed a $52 \%$ reduction in the hazard of on-treatment all-cause mortality (HR 0.48 ; $95 \%$ CI 0.27 to 0.85 ; $P=0.012$ ) but the analysis was post hoc, based on a safety endpoint and not a prespecified secondary clinical outcome. It was not powered to examine differences in mortality and had 
incomplete follow-up.

There is a post hoc, stratified safety pooled analysis of fatal adverse events in patients who participated in three 52-week trials: TRILOGY ( $\mathrm{N}=1367), \operatorname{TRINITY}(\mathrm{N}=2690)$ and TRIBUTE $(\mathrm{N}=1532) .{ }^{5}$ These studies compared a single inhaler triple (inhaled corticosteroid [ICS]/long-acting beta2-agonist [LABA]/long-acting muscarinic antagonist [LAMA]) combination of beclomethasone bipropionate (BDP) $100 \mu \mathrm{g} /$ formoterol fumarate (FORF) 6 $\mu \mathrm{g}$ (FORF)/glycopyrronium bromide(G) $12.5 \mu \mathrm{g}$ to either:

(1) BDP/FORF --TRILOGY study or

(2) tiotropium (TIO) and BDP/FORF+TIO (in separate inhaler)--TRINITY study or

(3) indacterol/G--TRIBUTE study

Patients had severe to very severe COPD and had to have at least 1 exacerbation in the previous year. The study demonstrated a numerically significant difference in risk of developing a fatal event (HR 0.71: $95 \%$ CI 0.50 to 1.02; $P=0.066$ ). Interestingly, the only statistically significant results in this study were for non-respiratory fatal events wherein the ICS-containing treatments compared to the ICS-free treatments demonstrated a significant benefit (HR: $0.65,95 \%$ CI: 0.43 to $0.97, p=0.037$ ). While the authors did not specify the nature of the non-respiratory fatal events, several other studies have demonstrated a reduction in cardiovascular-related deaths that has been similar to the reduction in respiratory-related deaths.

Further, a systematic review and Bayesian metaanalysis of 219 trials found that ICS/LAMA/LABA or ICS/LABA conferred a statistically significant survival benefit compared to placebo with (OR $=0.74,95 \% \mathrm{CI}$ 0.59 to 0.93$) P(O R>1)=0.004$ for ICS/LAMA/LABA; and for ICS/LABA (OR=0.86, 95\% CI 0.76 to 0.98 ) $P(\mathrm{OR}>1)=0.015^{6}$

While these trials have suggested that the use of ICSs may confer a survival benefit for patients with severe COPD, they have had design flaws such as being underpowered, having inappropriate patient selection criteria, having a small sample size, having a short study duration, doing a post hoc analysis without prespecifying mortality as an outcome, having incomplete follow-up of vital status of participants who withdraw and using safety event data rather than true mortality data. Fortunately, current investigators have been taking the lessons learned from these earlier studies to further refine their study design to properly address this very important question as to whether ICSs do indeed reduce the risk of allcause mortality compared with non-ICS-containing inhaled medications. In this Journal Club we will review the more recent InforMing the Pathway of Chronic Obstructive Pulmonary Disease Treatment (IMPACT) trial, ${ }^{7,8}$ and the Efficacy and Safety of Triple Therapy in Obstructive Lung Disease (ETHOS) trial $^{9,10}$ to understand the evolution of the study design to consider the mortality question over the past several years. Both of these studies present fairly compelling evidence that ICS-containing triple therapy formulations in patients with severe COPD and a history of exacerbations do indeed reduce the risk of all-cause mortality compared to long-acting dual bronchodilators.

\section{Abstract 1 Reduction in All-Cause Mortality with Fluticasone Furoate/Umeclidinium/ Vilanterol in Patients with Chronic Obstructive Pulmonary Disease}

Lipson DA, Crim C, Criner GJ, et al; IMPACT Investigators. Am J Respir Crit Care Med. 2020;201(12):15081516.

doi: https://doi.org/10.1164/rccm.201911-2207OC

Rationale: The IMPACT (Informing the Pathway of Chronic Obstructive Pulmonary Disease Treatment) trial demonstrated a significant reduction in allcause mortality (ACM) risk with fluticasone furoate/ umeclidinium/vilanterol (FF/UMEC/VI) versus $\mathrm{UMEC} / \mathrm{VI}$ in patients with chronic obstructive pulmonary disease (COPD) at risk of future exacerbations. Five hundred seventy-four patients were censored in the original analysis owing to incomplete vital status information.

Objectives: Report ACM and impact of stepping down therapy, following collection of additional vital status data.

Methods: Patients were randomized 2:2:1 to FF/ UMEC/VI 100/62.5/25 $\mu$, FF/VI $100 / 25 \mu \mathrm{g}$, or UMEC/VI $62.5 / 25 \mu \mathrm{g}$ following a run-in on their 
COPD therapies. Time to ACM was prespecified. Additional vital status data collection and subsequent analyses were performed post hoc.

Measurements and Main Results: We report vital status data for $99.6 \%$ of the intention-to-treat population $(n=10,355)$, documenting $98(2.36 \%)$ deaths on FF/UMEC/VI, 109 (2.64\%) on FF/VI, and 66 (3.19\%) on UMEC/VI. For FF/UMEC/VI, the hazard ratio for death was 0.72 (95\% confidence interval, 0.53-0.99; $P=0.042)$ versus UMEC/VI and 0.89 (95\% confidence interval, 0.67-1.16; $P=0.387$ ) versus $\mathrm{FF} / \mathrm{VI}$. Independent adjudication confirmed lower rates of cardiovascular and respiratory death and death associated with the patient's COPD.

Conclusions: In this secondary analysis of an efficacy outcome from the IMPACT trial, once-daily single-inhaler FF/UMEC/VI triple therapy reduced the risk of ACM versus UMEC/VI in patients with symptomatic COPD and a history of exacerbations.

\section{Comments}

The IMPACT trial was a 52-week study that had 10,335 patients and compared triple therapy (ICS/LAMA/ LABA) versus ICS/LABA and LAMA/LABA. ${ }^{7}$ The primary outcome was reduction in moderate to severe exacerbations, however, they also examined several secondary end points including all-cause mortality. Selection criteria for patients in the IMPACT trial differed from the TORCH and SUMMIT trials in that the patients had more severe disease and they had an exacerbation history of at least 1 moderate or severe (hospitalized) exacerbation if the forced expiratory volume in 1 second $\left(\mathrm{FEV}_{1}\right)$ was $<50 \%$ predicted, or 2 moderate or 1 severe exacerbation if the $\mathrm{FEV}_{1}$ was between $50 \%$ and $80 \%$ predicted. Patients with a current diagnosis of asthma were excluded. At trial entry, about $38 \%$ of the patients were already receiving triple therapy. Randomization was $2: 2: 1$ and the FF/umeclidinium(UMEC)/VI group had 4151 participants, the FF/VI group had 4134 participants and the UMEC/VI group had 2070 participants.

The study did indeed meet its primary endpoint showing a significant reduction in the rate of moderate to severe exacerbations for triple therapy (FF/UMEC/ VI) 0.91 /year versus (FF/VI) 1.07/year and (UMEC/ VI), 1.21/year with $P$ values for both comparisons being less than 0.001. The trial also demonstrated statistically significant beneficial outcomes for triple therapy compared to both dual therapy combinations with respect to hospitalizations, improved lung function and health-related quality of life.

Interestingly, patients who had eosinophil counts greater than 150 cells per microliter demonstrated a greater reduction in the exacerbation rate for the steroid-containing arms versus the LABA/LAMA arms. Among patients with eosinophil levels of less than 150 cells per microliter, the annual rate of moderate or severe exacerbations was 0.85 (95\% CI, 0.80 to 0.91 ) with FF/UMEC/VI, 1.06 (95\% CI, 0.99 to 1.14) with FF/VI, and 0.97 (95\% CI, 0.88 to 1.07 ) with UMEC/VI. Among patients with eosinophil levels of at least 150 cells per microliter, the annual rate was 0.95 (95\% CI, 0.90 to 1.01) with triple therapy, 1.08 (95\% CI, 1.02 to 1.14 ) with FF/VI, and 1.39 (95\% CI, 1.29 to 1.51) with UMEC/VI.

Death during treatment occurred in $1 \%$ of the $\mathrm{FF} /$ UMEC/VI group, $1 \%$ of the FF/VI group, and $2 \%$ of the UMEC/VI group. All-cause mortality was significantly lower with the regimens that included the inhaled FF compared to UMEC/VI. The hazard ratio for triple therapy versus UMEC/VI was 0.58 (95\% CI, 0.38 to $0.88 ; 42 \%$ difference; unadjusted $P=0.01)$, and the hazard ratio for FF/VI versus UMEC/VI was 0.61 (95\% CI, 0.40 to 0.93 ; $39 \%$ difference; unadjusted $P=0.02$ ).

An analysis of independently adjudicated specific causes of death during treatment revealed a lower rate of deaths from both cardiovascular and respiratory causes in the inhaled-glucocorticoid groups than in the UMEC/VI group. The rate of cardiovascular deaths on treatment per 1000 patient years was 4.2 (FF/UMEC/VI), 6.0 (FF/VI), and 8.7 (UMEC/VI). For deaths adjudicated to be related to respiratory causes the rate per 1000 patient years was 4.0, 3.4, and 5.2, respectively and for deaths adjudicated to be related to the patients' underlying COPD, the rate per 1000 patient years, was 4.8, 4.0, and 8.7, respectively.

Despite these results, the investigators were somewhat cautious in interpreting the all-cause mortality benefit given that 574 participants were censored in the original, week-52 analysis because of incomplete vital status data. The paper referenced above ${ }^{8}$ is the additional post hoc analysis that provided vital status at week 52 for $99.6 \%$ of the intent-totreat population with only 42 participants ultimately censored due to missing data. This added 27 additional 
off treatment deaths, 9 on FF/UMEC/VI, 12 on FF/VI and 6 on UMEC/VI. Combining these deaths with the on-treatment group demonstrated a HR for all-cause mortality of $0.72 \mathrm{FF} / \mathrm{UMEC} / \mathrm{VI}$ versus UMEC/VI (95\%CI, 0.53 to $0.99, P=0.042$ ) and HR of 0.82 for $\mathrm{FF} /$ VI versus UMEC/VI (95\% CI, 0.60 to $1.11, P=0.190$.) The results were essentially unchanged even when the first 30, 60 or 90 days of treatment were excluded in order to eliminate the possibility that the deaths may have occurred as a result of acute withdrawal of ICSs for patients who were stepped down to LAMA/LABA therapy.

To assess the robustness of the data, the investigators performed a tipping point analysis that demonstrated that if all patients on UMEC/VI who had censored data were entered as alive at the end of 52 weeks, the postwithdrawal hazard for FF/UMEC/VI would need to be approximately 10 times higher than the pre-withdrawal hazard before losing statistical significance. Also, if they assumed that the UMEC/VI group that had censored data had the same post-withdrawal and pre-withdrawal hazard rate, then the FF/UMEC/VI would need to be 14 times higher than the pre-withdrawal hazard before losing statistical significance. It was suggested that these extreme assumptions were unlikely and that the observed results were indeed robust to missing data.

There was a higher incidence of pneumonia in the ICS-containing groups FF/UMEC/VI and FF/VI (ICS/ LABA) than in the UMEC/VI dual bronchodilator group, $8 \%, 7 \%$ and $5 \%$ respectively. The hazard ratio comparing triple therapy to UMEC/VI was 1.53 (95\% confidence interval, 1.22 to $1.92 ; P<0.001$ ). A follow-up analysis by Dransfield and colleagues ${ }^{11}$ assessed the relative benefit of exacerbation reduction compared with the increased risk of pneumonia by evaluating time to first (prespecified) and rates (post hoc) of investigator-reported pneumonia, serious pneumonia (leading to hospitalization or death) and composite endpoints of: (1) moderate/severe exacerbations or pneumonia, and (2) severe exacerbation or serious pneumonia. Their analysis demonstrated that despite the ICS/LABA/LAMA being associated with a higher incidence of pneumonia, the combination still showed a favorable benefit risk profile of FF/UMEC/VI versus FF/VI (ICS/LABA) and UMEC/VI (LAMA/LABA) and a persistent overall benefit with the use of FF/UMEC/ VI compared to UMEC/VI.

\section{Abstract 2 Reduced All-Cause Mortality in the ETHOS Trial of Budesonide/ Glycopyrrolate/Formoterol for COPD: A Randomized, Double-Blind, Multi-Center Parallel-Group Study}

Martinez FJ, Rabe KF, Ferguson GI, et al; ETHOS investigators. Am J Respir Crit Care Med. Published online 2020 November 30.

doi: https://doi.org/10.1164/rccm.202006-26180C

Rationale: In the Phase III, 52-week ETHOS trial in COPD (NCT02465567), triple therapy with budesonide/glycopyrrolate/formoterol fumarate (BGF) significantly reduced all-cause mortality versus glycopyrrolate/formoterol fumarate (GFF). However, 384 out of 8,509 patients were missing vital status at Week 52 in the original analyses.

Objective: To assess the robustness of the ETHOS mortality findings following additional data retrieval for patients missing Week 52 vital status in the original analyses.

Methods: Patients with moderate-to-very severe COPD and prior history of an exacerbation received twice-daily dosing with BGF $320 / 18 / 9.6 \mu \mathrm{g}$ or

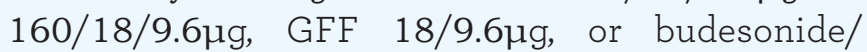
formoterol fumarate (BFF) 320/9.6 $\mathrm{g}$ (all delivered via a single metered dose Aerosphere inhaler). Time to death (all-cause) was a pre-specified secondary endpoint.

Measurements and Main Results: In the final retrieved dataset, which included Week 52 vital status for $99.6 \%$ of the intent-to-treat population, risk of death with BGF 320 was significantly lower versus GFF (hazard ratio [HR] 0.51, 95\% confidence interval [CI] 0.33-0.80; unadjusted $P=0.0035)$. There were no significant differences in mortality when comparing BGF 320 to BFF (HR 0.72, 95\% CI 0.44$1.16 ; P=0.1721$ ), nor were significant differences observed when comparing BGF 160 against either dual comparator. Results were similar when the first 30,60 , or 90 days of treatment were excluded from the analysis. Deaths from cardiovascular causes 
occurred in $0.5 \%, 0.8 \%, 1.4 \%$, and $0.5 \%$ of patients in the BGF 320, BGF 160, GFF, and BFF groups, respectively.

Conclusions: Using final retrieved vital status data, triple therapy with BGF 320/18/9.6ug reduced the risk of death compared with GFF but was not shown to significantly reduce the risk of death compared with BFF, in patients with COPD. Triple therapy containing a lower dose of inhaled corticosteroid (BGF 160/18/9.6ug) was not shown to significantly reduce risk of death compared with the dual therapy comparators.

\section{Comments}

The ETHOS trial ${ }^{10}$ studied single inhaler triple therapy at 2 different dose levels of the ICS, budesonide (BUD), and compared them with dual therapy combinations of the monocomponents: LAMA/LABA (G/FF) and the ICS/LABA combined. The primary endpoint was the rate of moderate to severe COPD exacerbations. Mortality was assessed as a prespecified secondary outcome. The primary paper notes that there was a statistically significant reduction in all-cause mortality for both triple and single inhaler formulations. The 2 budesonide dose levels were tested in order to judge the relative efficacy versus safety profile with a particular focus on pneumonia. Patients had to have moderate to severe COPD, post-bronchodilator $\mathrm{FEV}_{1}$ between 30\% and $65 \%$, (about $61 \%$ had an $\mathrm{FEV}_{1}<50 \%$ ), a COPD Assessment Test score of at least 10 and at least 1 exacerbation in the past year if the $\mathrm{FEV}_{1}$ was less than $50 \%$ or at least 2 moderate or 1 severe exacerbation if the $\mathrm{FEV}_{1}$ was greater than $50 \%$.

Both doses of the triple inhaler were superior to the LAMA/LABA (G/FF) and the ICS/LABA (BUD $320 \mu \mathrm{g} / \mathrm{FF} 9.6 \mu \mathrm{g})$ combinations in reducing exacerbations. There was no significant difference between the higher and lower ICS dose triple combinations in terms of reducing exacerbations. The $320 \mu \mathrm{g}$ BUD triple therapy compared to G/FF was $24 \%$ lower; (rate ratio 0.76 ; $95 \%$ CI 0.69 to 0.83 ; $p<0.001$ ) and compared to B/FF 13\% lower: (rate ratio, $0.87 ; 95 \% \mathrm{CI}, 0.790$ to $0.95 ; p=0.003$ ). For the BUD $160 \mu \mathrm{g}$ formulation the reduction compared to the G/FF was $25 \%$ lower: (rate ratio 0.86 ; $95 \% \mathrm{CI}, 0.69$ to $0.83 ; P<0.001$ ) and compared to $\mathrm{B} / \mathrm{FF}$ it was $14 \%$ lower: (rate ratio, 0.86 : $95 \% \mathrm{CI}, 0.79$ to $0.95 ; P=0.002$ ).

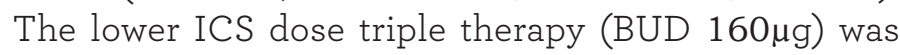
actually more efficacious in reducing exacerbations than the higher dose ICS/LABA combination of BUD $360 \mu \mathrm{g} / \mathrm{FF} 9 \mu \mathrm{g}$.

The incidence of confirmed pneumonia was from $3.5 \%$ to $4.5 \%$ in the groups that included inhaled glucocorticoids use and $2.3 \%$ in the G/FF group. BUD $/ 360 \mu$ g was $4.2 \%$, BUD/160 $\mu$ g $3.5 \%$, and BUD/ FF was $4.5 \%$.

The paper by Martinez and colleagues (abstract above) focused on the all-cause mortality. It was assessed as a prespecified secondary outcome. There were 8509 patients as part of a modified intentionto-treat group and 7187 patients completed the trial. Because of the importance of having final vital status data on all participants in the intention-to-treat group, it was critical to collect such information on as many participants as possible. There were 384 patients with missing vital status data at week 52 . In this follow-up paper they were able to retrieve vital status data at week 52 for all but 30 patients resulting in retrieval of vital status data for $99.6 \%$ of the intention-to-treat population. The results, as outlined in the abstract, demonstrate the risk of death for the BUD $320 \mu \mathrm{g} / \mathrm{G} /$ FF group (BGF320) was significantly lower compared to $G / F F$ with a $P$ value of 0.0035 and a number needed to treat of 80 (95\% CI 58 to 198). BGF320 did not significantly reduce the risk of death compared to BGF 160 or to B/FF320. BGF 160 showed trends of reduced risk of death compared to B/FF320 and G/FF but they were not statistically significant.

The investigators performed various analyses to assess the possible impact of the 30 patients with missing week 52 vital status data. These included tipping point analyses and landmark analyses. These analyses demonstrated the numerical benefits for time to death in favor of BFG320 versus dual therapies across all subgroups. Landmark analyses excluded deaths that occurred in the first 30,60 or 90 days of the study onset and ruled out the possible impact of acute (step down) ICS treatment withdrawal on the findings. The benefits of BGF320 were found to be greatest for those with at least 2 exacerbations in the previous year or a post-bronchodilator $\mathrm{FEV}_{1}$ at least $50 \%$ and for those with prior triple therapy or ICS use. Further, the benefit of BGF320 versus GFF in reducing mortality generally increased with eosinophil count.

Compared to patients who survived, the patients who 
died had a higher mean age, a lower mean FEV 1 (\% predicted and \% reversibility), and a higher proportion indicated prior ICS use. The association of prior ICS use here is more likely a reflection of the severity of their disease than some effect of withdrawing ICS at time of randomization.

ETHOS and IMPACT have similar designs in terms of patient selection characteristics, large sample size characteristics, duration of trial and comparison with regard to ICS/LAMA/LABA, ICS/LABA and LAMA/ LABA. ETHOS also had an ICS dose comparison for the single triple inhaler with a BUD $320 \mu \mathrm{g}$ and a $160 \mu \mathrm{g}$ dose level. Both studies used the same components and same delivery device for the triple inhaler or the dual combinations They also selected similar patients with moderate to severe COPD and a significant exacerbation history where frequency of exacerbation criteria differed according to pre-bronchodilator $\mathrm{FEV}_{1}$ (1 exacerbation if $\mathrm{FEV}_{1}$ was $<50 \%$ and 2 moderate or 1 severe exacerbation if the $\mathrm{FEV}_{1}$ was at least $50 \%$ predicted), unlike the TORCH and the SUMMIT trials. The ETHOS trial also only took patients with a postbronchodilator $\mathrm{FEV}_{1}<65 \%$ whereas IMPACT took participants with an $\mathrm{FEV}_{1}$ up to $80 \%$, however, the average $\mathrm{FEV}_{1}$ value in the ETHOS trial was $43.6 \%$ predicted and in the IMPACT trial $45 \%$ predicted. They both looked at all-cause mortality as a prespecified secondary outcome and were able to collect vital status data for over $99 \%$ of the participants in both of these post hoc analyses. Both of these studies demonstrate a statistically significant reduction in mortality in the intention-to-treat populations with over $99 \%$ of the participants included following rigorous retrieval of vital status data for patients who had not completed the full 52 weeks of the trial. The survival benefit has been shown to be quite robust and quite compelling and more than simply the result of deaths related to ICS withdrawal. The investigators utilized lessons learned from trials such as TORCH and SUMMIT and selected patients with more severe disease and/or significant exacerbation histories.

The studies both reinforce that the reduction in mortality is not just related to reducing exacerbations but also related to reductions in cardiovascularrelated deaths that represent a substantial portion of the deaths in COPD patients. The precise mechanism by which ICSs may reduce mortality remains unclear but various hypotheses commonly cited include reductions in hyperinflation that may improve cardiac function, improved oxygenation reducing coronary ischemia, suppression of aldosterone, and reductions in systemic inflammation. ${ }^{12}$

Both trials also showed that there was a correlation between higher peripheral blood eosinophil levels and reduced frequency of exacerbations and improved survival. This certainly supports that the survival benefit is related at least in part to the decreased steroid responsive inflammatory component. The ETHOS study is also instructive so far as the BUD320 $\mu \mathrm{g} / \mathrm{G} / \mathrm{FF}$ group showed statistically significant reductions in mortality while the $160 \mu \mathrm{g}$ dose group showed a trend but was not statistically significant indicating a dose response relationship. It is worth noting that the BUD $320 \mu \mathrm{g}$ dose is closer in potency to the FF $100 \mu \mathrm{g}$ dose used in IMPACT and the BDP $100 \mu \mathrm{g}$ dose used in the TRINITY, TRILOGY and TRIBUTE trials. ${ }^{5}$

There is also the suggestion from the ETHOS trial that there may be a survival benefit related to the LAMA as the BUD320/G/FF group showed a statistically significant reduction in all-cause mortality compared to ICS/LABA BUD320/FF. The UPLIFT trial had also suggested the possibility of a survival benefit related to the use of LAMA. ${ }^{13}$

\section{The Bottom Line}

1. In 2 large, double blind randomized control trials (IMPACT and ETHOS), compelling evidence is presented to indicate that single triple ICS/ LAMA/LABA combination therapy has been demonstrated to have an increased survival benefit compared to combination dual long-acting LAMA/ LABA bronchodilator therapy.

2. Patients most likely to benefit include those who have had at least 1 exacerbation in the previous year if they have an $\mathrm{FEV}_{1}$ of less than $50 \%$ predicted or have 2 moderate or 1 severe exacerbation in the past year if the $\mathrm{FEV}_{1}$ is at least $50 \%$ or above.

3. There is evidence of a loose association between higher eosinophil counts above 150cells/ $\mu \mathrm{L}$ survival and survival benefit with the use of ICSs.

4. Triple therapy (ICS/LABA/LAMA) appears to improve survival greater than ICS/LABA suggesting that the use of LAMA in conjunction with ICS/LABA/LAMA confers some further improvement in survival.

5. The results for the ETHOS trial suggest that there 
is a dose response relationship with regard to the ICS dose and survival benefit.

6. One needs to weigh the relative risks and benefits of higher dose ICSs and the attendant risk of pneumonia. 


\section{References}

1. Soto-Campos JG, Plaza V, Soriano JB, et al. Causes of death in asthma, COPD and non-respiratory hospitalized patients: a multicentric study. BMC Pulm Med. 2013;13:73.

doi: https://doi.org/10.1186/1471-2466-13-73

2. Calverley PM, Anderson JA, Bartolome C, et al. Salmeterol and fluticasone propionate and survival in chronic obstructive pulmonary disease. N Engl J Med. 2007;356(8):775-789. doi: https://doi.org/10.1056/NEJMoa063070

3. Vestbo J, Andersen JA, Brook RD, et al. Fluticasone furoate and vilanterol and survival in chronic obstructive pulmonary disease with heightened cardiovascular risk (SUMMIT): a double-blind randomised controlled trial. Lancet. 2016;387(10030):1817-1826. doi: https://doi.org/10.1016/S0140-6736(16)30069-1

4. Wedzicha JA, Calverley PMA, Seemungal TA, Hagan G, Ansari Z, Stockley RA. The prevention of chronic obstructive pulmonary disease exacerbations by salmeterol/fluticasone propionate or tiotropium bromide. Am J Respir Crit Care Med. 2008; 177(1):19-26.

doi: https://doi.org/10.1164/rccm.200707-973OC

5. Vestbo J, Fabbri L, Papi A, et al. Inhaled corticosteroid containing combinations and mortality in COPD. Eur Respir J. 2018;52(6). doi: https://doi.org/10.1183/13993003.01230-2018

6. Lee HW, Park J, Jo J, Jang EJ, Lee C-H. Comparisons of exacerbations and mortality among regular inhaled therapies for patients with stable chronic obstructive pulmonary disease: Systematic review and Bayesian network meta-analysis. PLoS Med. 2019;16(11): e 1002958.

doi: https://doi.org/10.1371/journal.pmed.1002958

7. Lipson DA, Barnhart F, Brealey N, et al. Once-daily single-inhaler triple versus dual therapy in patients with COPD. $N$ Engl $\mathrm{J}$ Med. 2018;378(18):1671-1680.

doi: https://doi.org/10.1056/NEJMoa1713901

8. Lipson, DA, Crim C, Criner GJ, et al. Reduction in all-cause mortality with fluticasone furoate/umeclidinium/vilanterol in patients with chronic obstructive pulmonary disease. $A m J$ Respir Crit Care Med. 2020;201(12): 1508-1516.

doi: https://doi.org/10.1164/rccm.201911-2207OC

9. Martinez FJ, Rabe KF, Ferguson GT, et al. Reduced all-cause mortality in the ETHOS trial of budesonide/glycopyrrolate/ formoterol for COPD: a randomized, double-blind, multi-center parallel-group study. Am J Respir Crit Care Med. Published online 2020 November 30.

doi: https://doi.org/10.1164/rccm.202006-26180C

10. Rabe KF, Martinez FJ, Ferguson GT, et al; ETHOS investigators. Triple inhaled therapy at two glucocorticoid doses in moderateto-very-severe COPD. N Engl J Med. 2020;383(1):35-48. doi: https://doi.org/10.1056/NEJMoa1916046 